\title{
Frequency-domain Technique to Measure the Inertial Response of Forward Stimulated Brillouin Scattering for Acoustic Impedance Sensing
}

\author{
Desmond M. Chow, Marcelo A. Soto, and Luc Thévenaz \\ EPFL Swiss Federal Institute of Technology, Institute of Electrical Engineering, SCI STI LT, \\ Station 11, CH-1015 Lausanne, Switzerland.
}

\begin{abstract}
A frequency-domain method is proposed to measure the inertial response of forward stimulated Brillouin scattering, so that the acoustic impedance of a liquid medium outside an uncoated $30 \mathrm{~m}$ standard single-mode fibre can be sensed. The proposed technique overcomes the linewidth distortion present in the time-domain approach due to Kerr effect and reduce time and complexity for data post-processing. Experimental results demonstrate that acoustic impedances of water and ethanol agree well with the expected values.
\end{abstract}

Keywords: Acousto-optic interaction, Forward Stimulated Brillouin Scattering, Optical Fibre Sensors

\section{INTRODUCTION}

Forward stimulated Brillouin scattering (FSBS) is an opto-acoustic effect involving interaction of light with transverse acoustic eigenmodes in an optical waveguide, which can be stimulated by electrostriction. The subject has been widely studied despite treated as a source of purturbation that limits long-range soliton transmission and generation of optical squeezed states in an optical fibre. ${ }^{1,2}$ Recently, Antman et al. have demonstrated measurement of liquid acoustic impedance using FSBS. ${ }^{3}$ By removing the acrylate coating of a standard singlemode fibre (SMF), transverse acoustic waves in the fibre cladding are allowed to interact directly with the fibre surroundings through reflection. Resembling a cavity ring-down technique, the acoustic decay rate gives the multipass phonon loss which could be retrieved to calculate the acoustic impedance of the surrounding liquid.

The FSBS response can be observed by measuring the refractive index modulation caused by electrostrictioninduced transverse acoustic waves. ${ }^{4}$ A high intensity short pulse approximating a delta function is launched into the fibre to generate multiple acoustic modes simultaneously, whilst an interferometer is used to convert optical phase changes in the fibre to intensity variations detectable by a photodiode. Although this time-domain technique proves the feasibility for acoustic impedance measurement, ${ }^{3}$ it retains a few practical drawbacks: 1 . Sophisticated data processing is required; the decay time of a particular mode must be obtained by applying Fourier transform numerically to the temporal trace and spectrally selecting a specific resonance peak. 2 . The non-ideal delta pulse used in the system has limited bandwidth, resulting in higher-order acoustic modes with reduced intensities. 3. The first few cycles of the time-domain trace are distorted due to the detrimental impact of Kerr effect, which must be eliminated manually.

In order to realize a precise and more direct measurement, a frequency sweeping method is preferable due to the flexibility in selecting the desired frequency band. A variant of FSBS frequency sweeping methods have been described previously. ${ }^{5-7}$ However, due to the presence of Kerr effect giving a similar signature in phase quadrature, the spectra of resonances are either distorted or must be manually processed to obtain an accurate decay time. Here, we demonstrate an acoustic wave frequency sweeping method by taking advantage of the contrast between the long inertial response of FSBS and the instantaneous nature of Kerr effect. FSBS is generated by a radio frequency $(\mathrm{RF})$ modulated optical pulse and interrogated with a Sagnac configuration. Yet, only the acoustic inertial response outside of the pulse duration is selectively gated for detection. The measured

Further author information: (Send correspondence to Desmond M. Chow)

Desmond M. Chow: E-mail: desmond.chow@epfl.ch, Telephone: +41 216937387

25th International Conference on Optical Fiber Sensors, edited by Youngjoo Chung, Wei Jin,

Byoungho Lee, John Canning, Kentaro Nakamura, Libo Yuan, Proc. of SPIE Vol. 10323,

1032311 · C 2017 SPIE · CCC code: 0277-786X/17/\$18 · doi: 10.1117/12.2265736

Proc. of SPIE Vol. $103231032311-1$ 
(a)

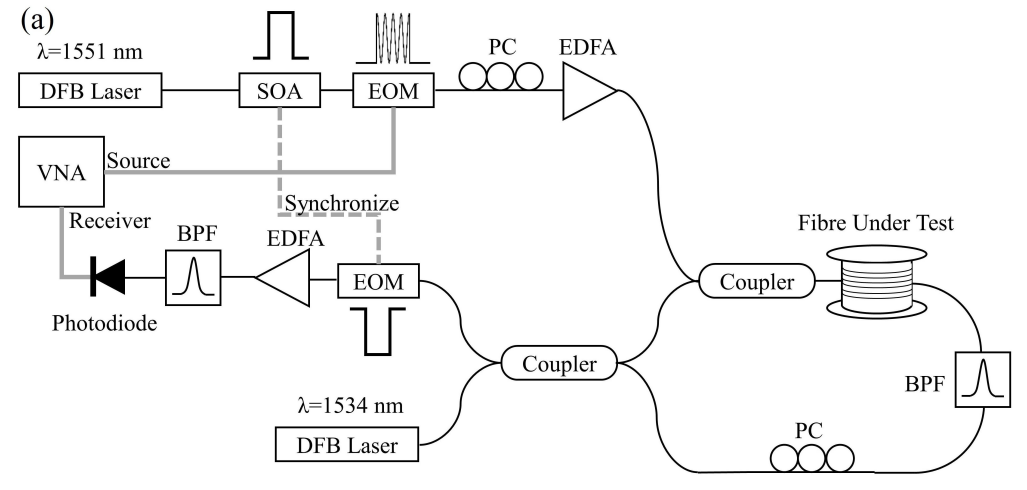

(b)

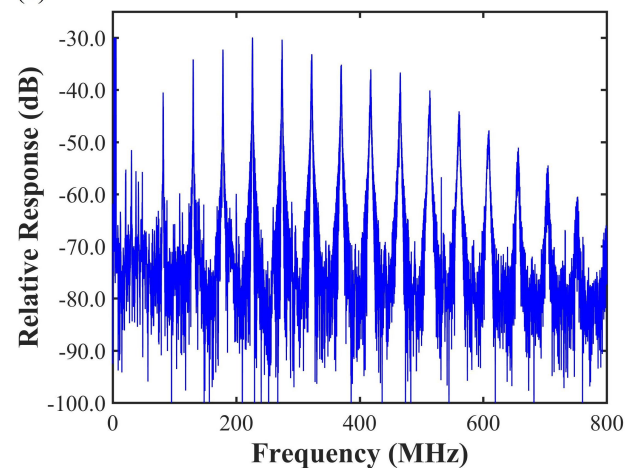

Figure 1. (a) Experimental setup of FSBS frequency-scan. (b) FSBS Spectrum of a 30 m uncoated SMF.

acoustic impedances of water and ethanol agree with the reported values. ${ }^{3}$ Measurement time is proportional to the scanning bandwidth and resonance linewidth could be read directly from the instrument without cumbersome numerical processing. The proposed technique validates the feasibility of frequency-scan in acoustic impedance measurement and could be valuable in the implementation of prospective distributed FSBS measurements.

\section{PRINCIPLE AND MEASUREMENT TECHNIQUE}

The acoustic wave guiding properties in SMF are based on the cylindrical shape of the cladding structure, which supports a discrete set of longitudinal, radial, torsional and flexural vibrations. FSBS is the interaction between light propagating inside the fibre core and the transverse acoustic eigenmodes within the cladding structure with close to zero longitudinal wavevector. The electrostriction-induced acoustic modes present in the forward light scattering are the radial modes $R_{0 m}$ and the mixed torsional-radial modes $T R_{2 m}$, which define acoustic waves that propagate radially and circumferentially, respectively. The characteristic solutions of $R_{0 m}$ modes, $y_{m}$, could be obtained from the equation. ${ }^{8}$

$$
\left(1-\alpha^{2}\right)\left(J_{0}\right)(y)-\alpha^{2} J_{2}(y)=0
$$

The cylindrical symmetry of the fibre results in solutions based on the Bessel functions $J_{n}$, where $\alpha$ is the ratio of the transverse acoustic velocity $V_{s}$ to the longitudinal acoustic velocity $V_{d}$ (e.g. $V_{d}=5968 \mathrm{~ms}^{-1}$ and $V_{s}=3764$ $m s^{-1}$ for fused silica). The eigenfrequencies $f_{m}$ of the $R_{0 m}$ modes are given by

$$
f_{m}=V_{d} y_{m} /(2 \pi a)
$$

where $a$ is the optical fibre radius. Details of FSBS mathematical model could be found in the literature. ${ }^{8,9}$

Due to photoelastic effect, the induced acoustic modes give rise to small refractive index change in the fibre core which generates pure phase-modulation that could be probed using an interferometer. We use the widely-proven technique of a Sagnac loop with continuous wave to retrieve the acoustic wave response. ${ }^{3,4}$ The experimental setup for frequency-scan of FSBS inertial response is shown in Fig. 1(a). A modulated optical pulse at $\lambda=1551 \mathrm{~nm}$ serves as the pump source for FSBS. A pulse of $50 \mathrm{~ns}$ is shaped from the incoming distributed feedback (DFB) laser by a semiconductor optical amplifier (SOA) and subsequently modulated by an electrooptic modulator (EOM) with RF frequency given by the source of a vector network analyser (VNA). The optical pulse is amplified by an Erbium doped fibre amplifier (EDFA) to produce a peak power of around $50 \mathrm{~mW}$. The pulse duration is optimized for the highest peak power without losing the strength of acoustic response. A continuous wave, from a second DFB laser at the well distinct wavelength of $1534 \mathrm{~nm}$, is used for probing the induced phase modulation. A tunable bandpass filter (BPF) adjusted to $1534 \mathrm{~nm}$ is placed in the Sagnac loop after the fibre under test (FUT) to prevent pump wave from travelling in the rest of the loop, whilst another $\mathrm{BPF}$ at the same wavelength is placed before the photodetector to avoid the pump wave from being detected. Knowing that the response of Kerr effect is instantaneous, the output trace is synchronously gated, blocking the part of the trace co-existing with the optical pulse (i.e. where Kerr effect is present) and leaving only the acoustic 
inertial response. The detected signal is read by the VNA synchronized to the RF modulation of the pump pulse. The FSBS spectrum of a $30 \mathrm{~m}$ uncoated SMF obtained from a VNA frequency sweep is shown in Fig. 1(b). The resonance peaks are clearly visible up to $800 \mathrm{MHz}$ since there is no limit in the pump pulse modulation bandwidth. The fading of higher-order resonance peaks is purely due to the growing mismatch between the field distributions of optical and acoustic modes.

\section{RESULTS AND DISCUSSIONS}

The resonance peak distortion caused by Kerr-induced phase-shift has been recently studied in the literature. ${ }^{5,6}$ The non-symmetric resonance spectrum observed in Fig 2(c) is due to the combine effect of cross-phase modulation and FSBS, as they have $\pi / 2$ phase difference with respect to each other. Numerically eliminating Kerr effect from the spectrum needs prior knowledge of the fibre nonlinear parameter, and therefore the analysis could be complicated for different fibres or pump conditions. Hereafter, the effectiveness of the proposed pulse gating method is demonstrated. Temporal traces and the corresponding VNA spectra of the detected response without and with pulse gating are compared. Without pulse gating, non-symmetric resonance spectra are observed (Fig $2(\mathrm{c}))$. By applying pulse gating, only the inertial acoustic response is detected, eliminating Kerr contribution (compare Fig 2(a) and (b)) and the resonance linewidth could be retrieved without ambiguity (Fig 2(d)).

The acoustic impedance of the detected medium is retrieved by finding the acoustic reflectivity at the claddingsurroundings boundary. ${ }^{3}$ Because of the large acoustic impedance mismatch between fibre material (silica) and the external medium, part of the transverse acoustic waves are reflected back and trapped in the transversal cavity. The intensity of subsequent oscillation cycles reduces exponentially in time due to reflection loss at the fibre boundary and internal loss (dissipation, structural inhomogeneity, etc.). The reflectivity coefficient, which depends on the acoustic impedance mismatch between silica and the surrounding medium, is given by ${ }^{3}$

$$
\left|r_{m i r}\right|=\left|Z_{f}-Z_{o}\right| /\left(Z_{f}+Z_{o}\right)
$$

where $Z_{f}$ and $Z_{o}$ are acoustic impedances of silica and surrounding medium, respectively. The resonance linewidth is the linear sum of linewidth broadening due to intrinsic loss and the intensity decay given by non-unity reflectivity at the boundary.

$$
\Delta v_{m}=\Delta v_{i n t}+\left(\Delta v_{i} / \pi\right) \ln \left(1 /\left|r_{m i r}\right|\right)
$$
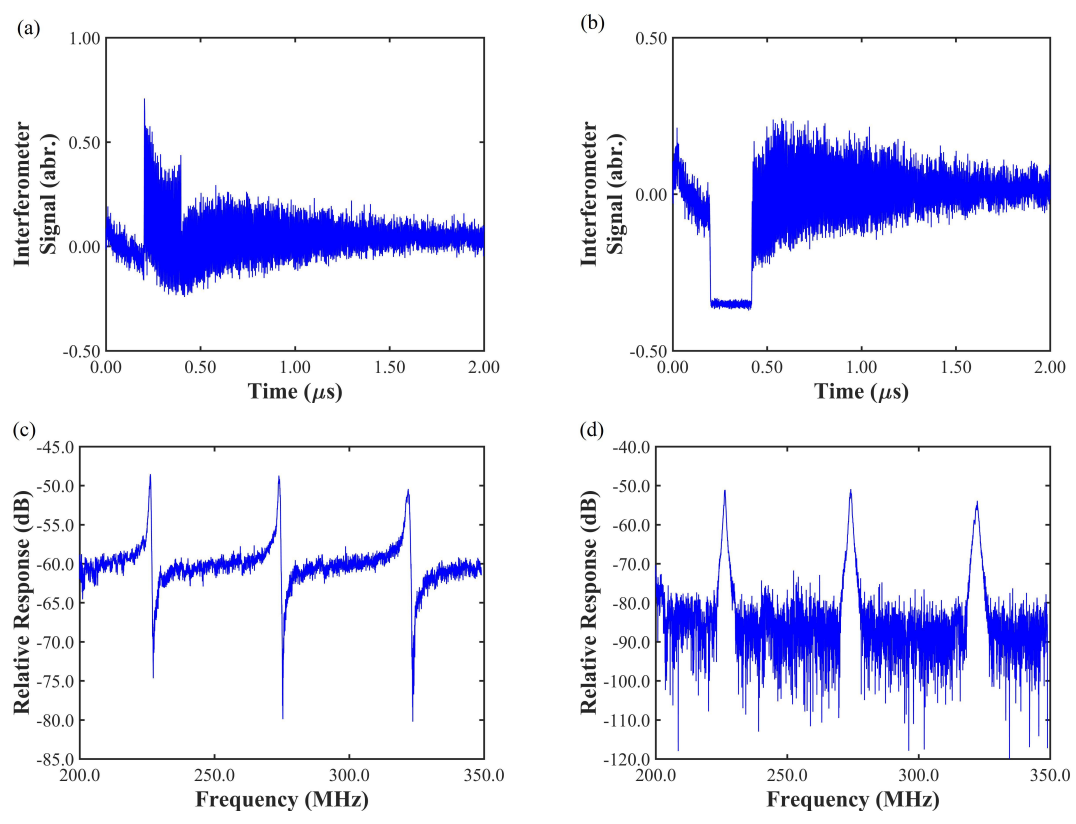

Figure 2. Temporal traces and the corresponding VNA spectra of the detected signal at photodetector (a)(c) without and $(b)(d)$ with pulse gating. 

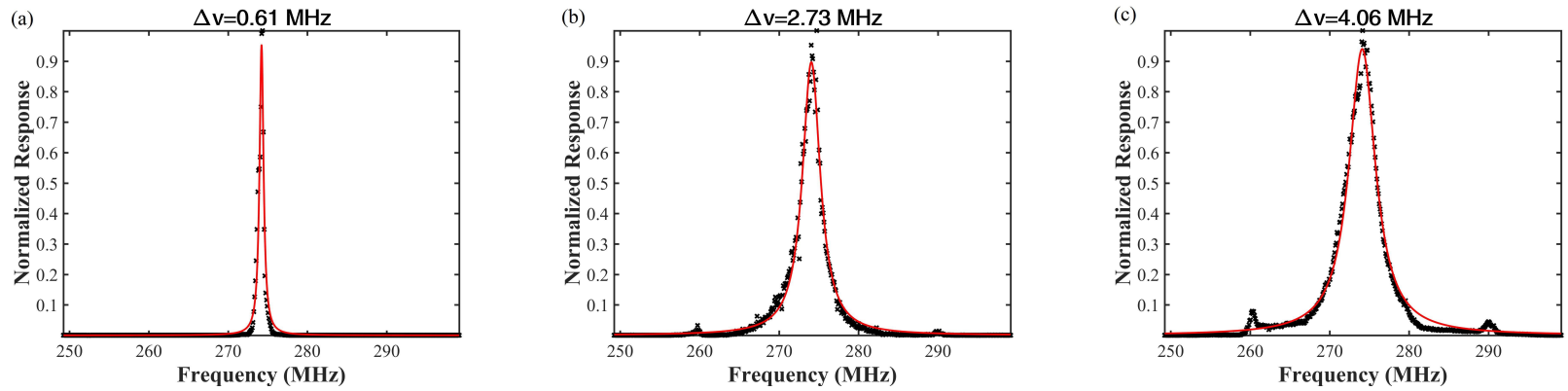

Figure 3. FSBS resonance linewidth with fibre in (a) air, (b) ethanol and (c) water.

where $\Delta v_{m}, \Delta v_{i n t}$ and $\Delta v_{i}$ are the linewidth of a measurement with a surrounding liquid medium, linewidth due to intrinsic loss (air as surroundings) and frequency separation between peaks, respectively. Results of the acoustic resonance linewidth measurements in air, water and ethanol are shown in Fig. 3. From the linewidth measurements, acoustic impedances of ethanol and water are $0.914 \mathrm{e} 6 \mathrm{~kg} / \mathrm{m}^{2} . s$ and $1.484 \mathrm{e} 6 \mathrm{~kg} / \mathrm{m}^{2} . s$, respectively, which agree with the reported values of $0.95 \mathrm{e} 6 \mathrm{~kg} / \mathrm{m}^{2} . s$ and $1.475 \mathrm{e} 6 \mathrm{~kg} / \mathrm{m}^{2} . s$, respectively. ${ }^{3}$

\section{CONCLUSION}

Acoustic impedance sensing has been demonstrated by frequency scanning of the FSBS inertial response, achieved by generating FSBS using optical pulse and gating the temporal trace of a Sagnac interrogation signal. The proposed method validates the feasibility of using frequency-domain approach to retrieve the acoustic impedance and eliminating the detrimental Kerr effect contribution. Furthermore, the technique simplifies data processing in such measurements, while benefiting from the intrinsic high stability and accuracy of frequency-domain techniques and opening potential possibilities for distributed mapping of FSBS to be investigated.

\section{ACKNOWLEDGEMENTS}

This work was supported by SNSF-ANR OASIS Grant 200021L-157132.

\section{REFERENCES}

[1] Le Quang, D., Jaoun, Y., Zimmerli, M., Gallion, P., and Thomine, J., "Time-resolved measurement of dynamic frequency chirp due to electrostriction mechanism in optical fibers," IEEE Photonics Technology Letters 8(3), 414-416 (1996).

[2] Poustie, A. J., "Guided acoustic-wave Brillouin scattering with optical pulses," Optics Letters 17(8), 574-576 (1992).

[3] Antman, Y., Clain, A., London, Y., and Zadok, A., "Optomechanical sensing of liquids outside standard fibers using forward stimulated Brillouin scattering," Optica 3(5), 510-516 (2016).

[4] Townsend, P. D., Poustie, A. J., Hardman, P., and Blow, K., "Measurement of the refractive-index modulation generated by electrostriction-induced acoustic waves in optical fibers," Optics Letters 21(5), 333-335 (1996).

[5] Wang, J., Zhu, Y., Zhang, R., and Gauthier, D. J., "FSBS resonances observed in a standard highly nonlinear fiber," Optics Express 19(6), 5339-5349 (2011).

[6] Renninger, W., Shin, H., Behunin, R., Kharel, P., Kittlaus, E., and Rakich, P., "Forward Brillouin scattering in hollow-core photonic bandgap fibers," New Journal of Physics 18(2), 025008 (2016).

[7] Antman, Y., London, Y., and Zadok, A., "Scanning-free characterization of temperature dependence of forward stimulated Brillouin scattering resonances," in [International Conference on Optical Fibre Sensors (OFS24)], 96345C-96345C, International Society for Optics and Photonics (2015).

[8] Poustie, A. J., "Bandwidth and mode intensities of guided acoustic-wave Brillouin scattering in optical fibers," JOSA B 10(4), 691-696 (1993).

[9] Biryukov, A., Sukharev, M. E., and Dianov, E. M., "Excitation of sound waves upon propagation of laser pulses in optical fibres," Quantum Electronics 32(9), 765 (2002). 